\title{
Inteligencia emocional y rendimiento académico en estudiantes de la Facultad de Educación y Humanidades de la Universidad Alas Peruanas
}

\author{
Emotional Intelligence and Academic Achievement \\ Students from the Faculty of Humanities and \\ Educaction at the University Alas Peruanas
}

Freddy Roberpierre Jaimes Álvarez*

http://dx.doi.org/10.21503/CienciayDesarrollo.2008.v9.02

\section{RESUMEN}

Con la presente investigación se busca hallar la relación que existe entre la inteligencia emocional y el rendimiento académico en estudiantes de la Facultad de Educación y Humanidades de la Universidad Alas Peruanas. Para ello, se han considerado cuatro componentes de la inteligencia emocional: el manejo del estado de ánimo, el manejo del estrés, la adaptabilidad, el componente emocional interpersonal y el componente emocional intrapersonal. La investigación es de tipo descriptivo-explicativo, pues realiza mediciones de las variables inteligencia emocional y rendimiento académico, a fin de describir las relaciones entre ellas en un determinado momento. El método asumido y las técnicas de investigación fueron aplicadas a una población compuesta por estudiantes de la Facultad de Educación en los diversos períodos académicos.

Palabras clave: Inteligencia emocional, estrés, adaptabilidad.

\section{ABSTRACT}

With the present investigation it is looked for to find the relationship that exists between the emotional intelligence and the academic yield in students of the Ability of Education and Humanities of the University Peruvian Wings. For it, they have been considered four components of the emotional intelligence: the handling of the state of spirit, the handling of the stress, the adaptability, the emotional interpersonal component and the component emotional intrapersonal. The investigation is of descriptiveexplanatory type, because it carries out mensurations of the emotional variable intelligence and academic yield, in order to describe the relationships among them in a certain moment. The assumed method and the investigation techniques were applied a population composed by students of the Ability of Education in the diverse academic periods.

Key words: Emotional intelligence, stress, adaptability.

\footnotetext{
* Docente de la Universidad Alas Peruanas.
} 


\section{INTRODUCCIÓN}

A pesar del desarrollo tecnológico y científico -especialmente del último siglo-, el hombre sigue siendo el mismo en términos de desarrollo evolutivo, es decir, las estructuras biológicas que regulan su comportamiento no han sufrido mayores cambios desde que terminaron las últimas glaciaciones, hace miles de años. Antes que el pensamiento o cognición existió la emoción, antes que la neocorteza -que nos hace Homo sapiens- existió el cerebro límbico. Tal vez una buena parte del sufrimiento humano pueda ser explicado desde esta nueva perspectiva que considera los nuevos hallazgos acerca del hombre desde la neurofisiología, la genética, la psicología cognitiva y la psicología del desarrollo. El concepto de inteligencia emocional es un intento, desde la ciencia, de explicar y resolver aquellas "inconsistencias" que son parte de la vida del hombre moderno.

Siempre se ha valorado la inteligencia como aquella característica distintiva de los seres humanos con respecto de los demás seres vivos, y, efectivamente, nuestra historia está llena de ejemplos en los que la solución a un problema se encontraba en la previa reflexión y en la aplicación creativa.

Sin embargo, paradójicamente, definir qué es la inteligencia es un problema que perdura hasta hoy. Las concepciones abarcan desde aquellas que la identifican como un "don especial" con el que algunos individuos nacen -se subrayan factores innatos o no aprendidos-, hasta aquellas que resaltan los factores sociales o medioambientales; y también las hay desdelas que sostienen la existencia de un solo factor " $G$ " de inteligencia - que subyace en el comportamiento "inteligente"-, hasta aquellas que proponen la existencia de más de un factor o tipos específicos de inteligencia.

El concepto de la inteligencia emocional ofrece un discurso coherente y brillantemente sustentado acerca de las "inconsistencias" mencionadas líneas arriba, cuando frente al aparente desarrollo científico-técnico el sufrimiento humano provenía de sí mismo, es decir, de la escasa comprensión y casi nula capacidad para controlar aquel comportamiento que lo conduce en muchas ocasiones a su autodestrucción.

La inteligencia emocional es un constructo teórico relativamente nuevo, pero de innegable importancia e impacto en la educación; me refiero al revolucionario concepto de la inteligencia emocional, anticipado por Howard Gardner(1) en su famosa teoría de "Las inteligencias múltiples". Es, pues, un constructo teórico que parte de la moderna investigación de la corriente cognitiva en la psicología, y, por otro lado, de las investigaciones de la biología del comportamiento humano. El término fue acuñado por Peter Salovey y John Mayer,(2) y popularizado por Daniel Goleman(3).

Sobre la inteligencia emocional se han realizado múltiples investigaciones, tanto desde la perspectiva de la investigación básica como aplicada en los diferentes campos del quehacer humano. Es muy difícil encontrar ahora algún profesional del campo de la educación que dude acerca de la importancia de la teoría de Gardner, y esta situación se explica por la difusión que se ha hecho de la aplicación práctica de esta teoría, la misma que, siendo relativamente nueva, es apoyada y confirmada constantemente por la praxis en diversos centros de enseñanza de todos los niveles.

Algo muy similar sucede con la inteligencia emocional. En nuestro país, esta teoría no es de larga data; sin embargo, a pesar de que ya se encuentra en el vocabulario del ciudadano promedio gracias a una amplia difusión de $L a$ inteligencia emocional de Daniel Goleman(3), es a veces difícil encontrar en la mayoría de las bibliotecas y librerías otros textos distintos 
al mencionado. Quizás la causa esté en la escasa investigación que existe al respecto. La información que llega frecuentemente es del tipo terapéutica, de autoayuda o como la descripción de una serie de competencias o habilidades que son apreciadas en determinados campos (laboral, organizacional, educativo, etc.).

La presente investigación tiene la finalidad de contribuir al desarrollo de esta línea de trabajo con la intención de proporcionar nuevos conocimientos que sirvan de base empírica no solo a sus presupuestos teóricos sino también a la praxis, que nunca debe dejarse en segundo lugar.

\section{Objetivos de la investigación}

\section{Objetivo general}

Determinar el grado de relación existente entre la inteligencia emocional y el rendimiento académico en estudiantes de la Facultad de Educación y Humanidades de la Universidad Alas Peruanas.

\section{Objetivos especificos}

- Determinar la relación entre el nivel del componente emocional del estado de ánimo general y el rendimiento académico en estudiantes de la Facultad de Educación y Humanidades de la Universidad Alas Peruanas.

- Determinar la relación entre el nivel del componente emocional de manejo del estrés y el rendimiento académico en estudiantes de la Facultad de Educación y Humanidades de la Universidad Alas Peruanas.

- Determinar la relación entre el nivel del componente emocional de adaptabilidad y el rendimiento académico en estudiantes de la Facultad de Educación y Humanidades de la Universidad Alas Peruanas.
- Determinar la relación entre el nivel del componente emocional interpersonal y el rendimiento académico en estudiantes de la Facultad de Educación y Humanidades de la Universidad Alas Peruanas.

- Determinar la relación entre el nivel del componente emocional intrapersonal y el rendimiento académico en estudiantes de la Facultad de Educación y Humanidades de la Universidad Alas Peruanas.

\section{Hipótesis}

\section{Hipótesis general}

Existe relación entre la inteligencia emocional y el rendimiento académico en estudiantes de la Facultad de Educación y Humanidades de la Universidad Alas Peruanas.

\section{Hipótesis especificas}

H1: El nivel del componente emocional del estado de ánimo está relacionado con el rendimiento académico de los estudiantes de la Facultad de Educación y Humanidades de la Universidad Alas Peruanas.

H2: El nivel del componente emocional de manejo del estrés está relacionado con el rendimiento académico de los estudiantes de la Facultad de Educación y Humanidades de la Universidad Alas Peruanas.

H3: El nivel del componente emocional de adaptabilidad está relacionado con el rendimiento académico de los estudiantes de la Facultad de Educación y Humanidades de la Universidad Alas Peruanas.

H4: El nivel del componente emocional interpersonal está relacionado con el rendimiento académico de los estudiantes de la Facultad de Educación y Humanidades de la Universidad Alas Peruanas. 
H5: El nivel del componente emocional intrapersonal está relacionado con el rendimiento académico de los estudiantes de la Facultad de Educación y Humanidades de la Universidad Alas Peruanas.

\section{MATERIAL Y MÉTODO}

\section{Tipo de investigación}

La presente investigación es de tipo descriptivo-explicativo, porque se realizan mediciones acerca de las variables: inteligencia emocional y rendimiento académico.

\section{Diseño de investigación}

El diseño de la presente investigación es transeccional correlacional porque se describen relaciones entre dos variables en un momento determinado.

\section{Población y muestra}

- Población: La población objeto de estudio estuvo compuesta por estudiantes de la Facultad deEducación enlos diversosciclos, de acuerdo a la siguiente distribución:

\begin{tabular}{|c|c|}
\hline CICLO & ALUMNOS \\
\hline Sexto & 33 \\
\hline Quinto & 35 \\
\hline Cuarto & 28 \\
\hline Tercero & 42 \\
\hline Segundo & 36 \\
\hline Primero & 54 \\
\hline TOTAL & 228 \\
\hline
\end{tabular}

- Muestra: Para determinar el tamaño óptimo de muestra, se utilizó la fórmula del muestreo aleatorio simple, la misma que se presenta a continuación:

$$
\mathrm{n}=\frac{\mathrm{Z}^{2} \mathrm{PQN}}{\mathrm{e}^{2}(\mathrm{~N}-1)+\mathrm{Z}^{2} \mathrm{PQ}}
$$

Donde:

$\mathrm{Z}=$ Valor de la abscisa de la curva normal para una probabilidad del $95 \%$ de confianza.

$\mathrm{P}=$ Proporción de estudiantes que presenta una buena inteligencia emocional $(\mathrm{P}=$ $0,5)$.

$\mathrm{Q}=$ Proporción de estudiantes que no presentan una buena inteligencia emocional $(\mathrm{Q}$ $=0,5)$.

$0 \%=$ Nivel de significancia 0,05 .

$\mathrm{e} \quad=$ error muestral $8 \%$.

$\mathrm{n}$ = Tamaño óptimo de la muestra.

Entonces, con un nivel de confianza del 95\% y $8 \%$ como margen de error muestral tenemos:

$$
\mathrm{n}=\frac{(1,96)^{2}(0,5)(0,5)(228)}{(0,08)^{2}(228-1)+(1,96)^{2}(0,5)(0,5)}
$$

$\mathrm{n}=91$ estudiantes

La muestra se elegirá al azar con el fin de minimizar posibles sesgos al obtener los resultados finales.

Utilizando la afijación proporcional, tenemos que, para cada ciclo, será seleccionado aleatoriamente:

$$
\mathrm{nh}=(\mathrm{n} / \mathrm{N}) \mathrm{Nh}
$$

Donde:

$\mathrm{Nh}=$ Población en cada ciclo $\mathrm{nh}=$ Muestra en cada ciclo.

$\mathrm{n} 1=91 / 228(54)=22, \quad \mathrm{n} 2=91 / 228(36)=$ $14, \mathrm{n} 3=91 / 228(42)=17 ; \mathrm{n} 4=91 / 228(28)$ $=11, \mathrm{n} 5=91 / 228(35)=14, \quad \mathrm{n} 6=91 / 228$ $(33)=13$ 


\begin{tabular}{|c|c|}
\hline CICLO & $\begin{array}{c}\text { MUESTRA DE } \\
\text { ALUMNOS }\end{array}$ \\
\hline Sexto & 13 \\
\hline Quinto & 14 \\
\hline Cuarto & 11 \\
\hline Tercero & 17 \\
\hline Segundo & 14 \\
\hline Primero & 22 \\
\hline TOTAL & 91 \\
\hline
\end{tabular}

\section{Técnicas e instrumentos de investigación}

\section{Técnicas}

- Análisis de documentos.

- Psicométrica.

- Análisis y procesamiento de datos.

\section{Instrumentos}

- Inventario de la inteligencia emocional a través del inventario de Bar-On (I-CE), creado por Reuven Bar- $\mathrm{On}^{6}$ para medir la inteligencia emocional, aplicable a sujetos de 16 años a más, de administración individual y/o colectiva. Adaptada a nuestro medio por la Dra. Nelly Ugarriza Chávez (baremos peruanos). Mediante 133 preguntas se evalúan 5 principales componentes conceptuales de la IE y social, los mismos que a su vez incluyen 15 factores.

A. Componente intrapersonal

1. Comprensión emocional de sí mismo.

2. Asertividad.

3. Autoconcepto.

4. Autorrealización.

5. Independencia.

B. Componente interpersonal

6. Empatía.

7. Relaciones interpersonales.

8. Responsabilidad social.
C. Componente de adaptabilidad

9. Solución de problemas.

10. Prueba de la realidad.

11. Flexibilidad.

D. Componente del manejo del estrés

12. Tolerancia al estrés.

13. Control de impulsos.

E. Componente del estado de ánimo general

14. Felicidad.

15. Optimismo.

\section{Pautas para interpretar los puntajes estándares del I-CE Bar-On}

\section{PUNTAJES \\ ESTÁNDARES PAUTAS DE INTERPRETACIÓN \\ 130 y más Capacidad emocional muy desarro- llada: marcadamente emocional. Ca- pacidad emocional inusual.}

116-129 Capacidad emocional muy desarrollada: alta, buena.

85-115 Capacidad emocional adecuada: promedio.

70 - 84 Necesita mejorar: baja. Capacidad emocional por debajo del promedio.

69 y menos Necesita mejorar: muy baja. Capacidad emocional extremadamente por debajo del promedio.

- Instrumento de recolección de notas / promedios: para la presente investigación se utilizarán los promedios ponderados finales del primer ciclo.

Técnicas de análisis y prueba de los resultados

\section{Determinación}

Relación Relación

Mediante el análisis de varianza (ANOVA) 


\section{RESULTADOS}

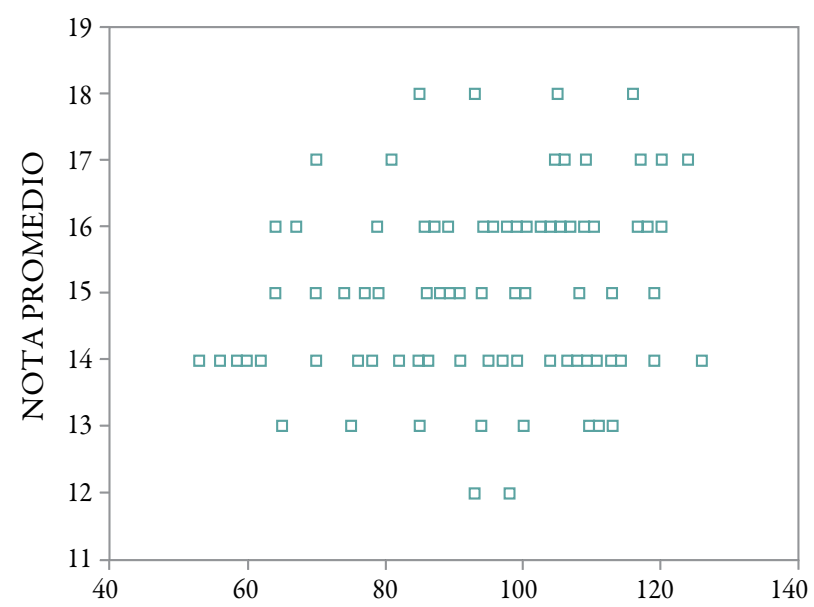

Cociente emocional del estado de ánimo general (CEAG) y rendimiento académico.

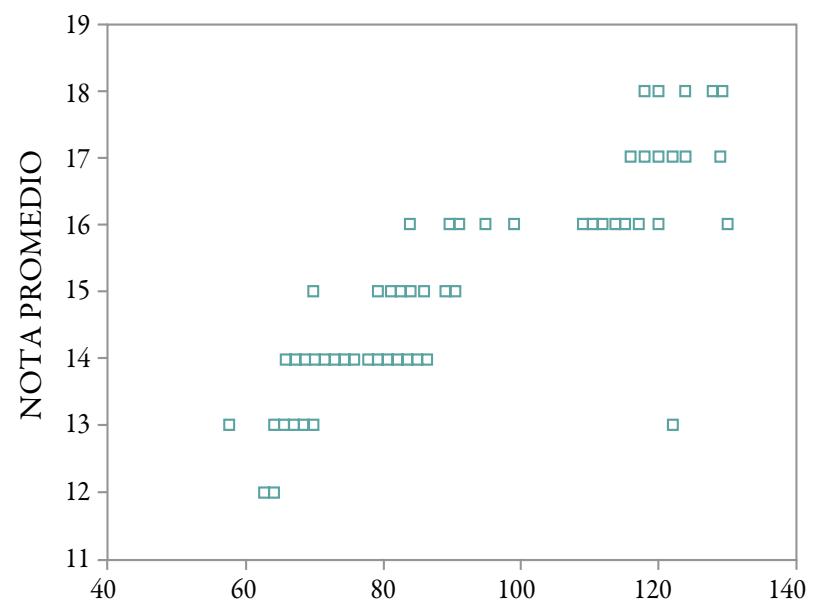

Cociente emocional del manejo del estrés (CEME) y rendimiento académico.

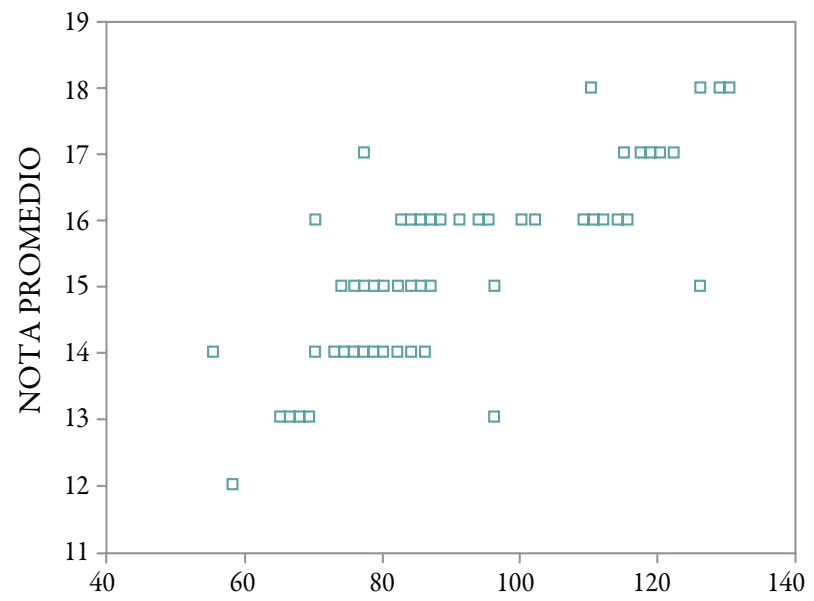

Cociente emocional de adaptabilidad (CEAD) y rendimiento académico.
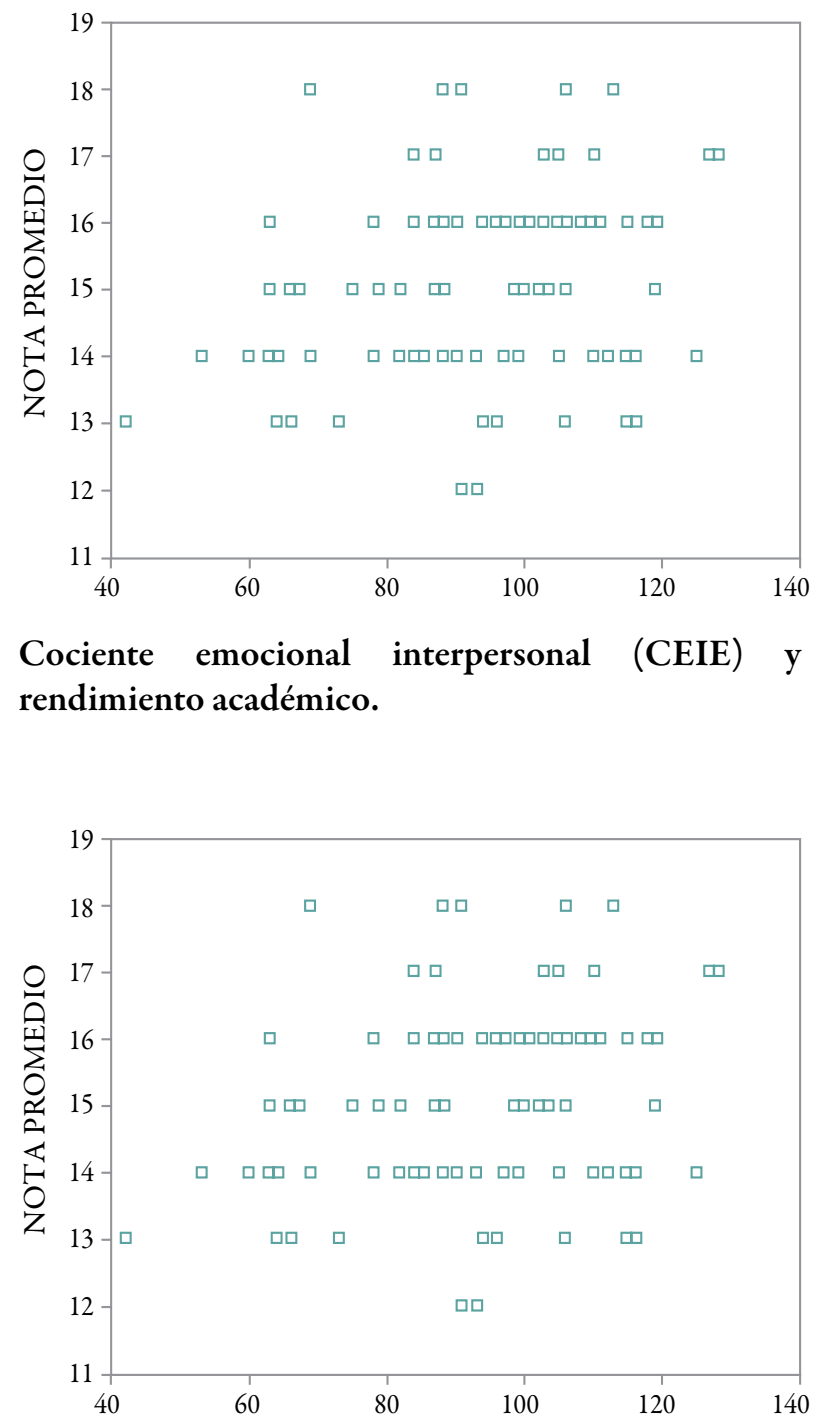

Cociente emocional intrapersonal (CEIA) y rendimiento académico.

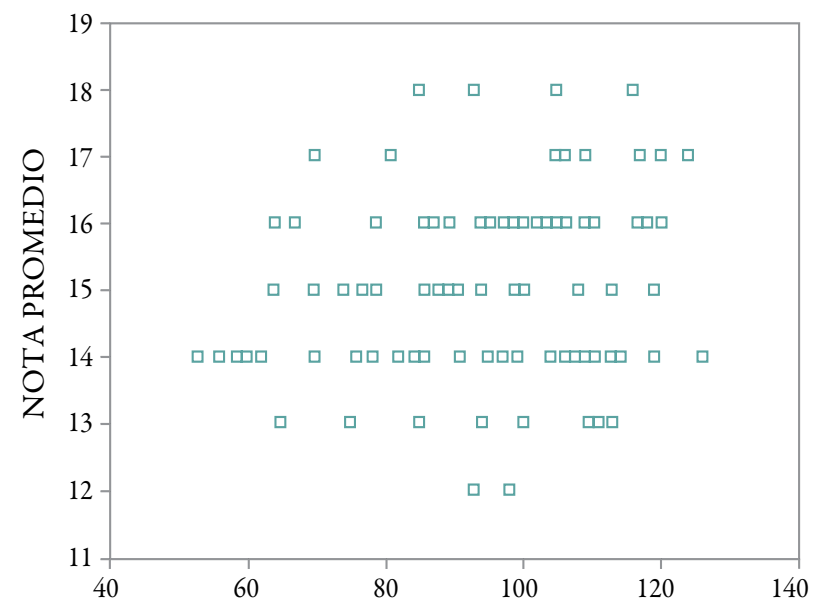

Cociente emocional total y rendimiento académico. 


\section{DISCUSIÓN}

En función de los resultados presentados, se concluye que no hay una relación significativa entre inteligencia emocional y rendimiento académico en los estudiantes de la Facultad de Educación y Humanidades de la Universidad Alas Peruanas.

Lo anterior se debe a que la variable dependiente, rendimiento académico, es una variable de índole racional o cognitiva si se quiere, mientras que lo que mide el inventario de Bar-On es el cociente emocional (CE), en otras palabras: inteligencia no cognitiva.

Como se ha señalado en el marco teórico, el rendimiento académico en nuestro sistema educativo consiste en aquella calificación vigesimal a cargo del profesor de turno, la misma que es el resultado de todo un proceso que empieza el primer día de clases, pero que siempre se inclina hacia la capacidad memorística y racional del alumno; esto quiere decir que aquel alumno que en una prueba responde lo más cerca de la información escrita de una separata o texto es el "mejor alumno", o, en otras palabras, que aquel alumno con las más altas calificaciones será el "mejor" del salón y por lo tanto le esperará una vida llena de "éxito".

Lo anterior, felizmente, ya ha sido descartado por Daniel Goleman, ${ }^{3}$ que luego de numerosos estudios concluye que aquellos llamados "genios" del salón de clases de la escuela o universidad no lo fueron tanto en la vida profesional, familiar o de pareja. Por tanto, no resulta extraño que estas variables no se encuentren en relación, y más bien nos confirman la idea de que aquello que llamamos rendimiento académico no abarca todas estas realidades de la naturaleza humana, como son la emoción, el sentimiento, la motivación, la adaptación, el manejo del estrés, el autoconocimiento y otros. Entonces es hora de ajustar y mejorar nuestra manera de hacer juicios sobre cuánto han aprendido verdaderamente nuestros alumnos, más aún si estos estudian para seguir nuestros pasos en la docencia.

Con respecto del componente emocional estado de animo, sí se encontró una relación significativa con la variable rendimiento académico, esto debido a que para rendir una prueba o superar cualquier reto, académico o no, es importante cierto grado de optimismo frente a la vida.

Lo mismo sucede con el componente emocional del manejo del estrés, donde se encontró una estrecha relación con la variable rendimiento académico, lo cual significa que a mayor capacidad de autocontrol, mayor es la posibilidad de resolver problemas.

El componente emocional de adaptabilidad sigue la tendencia de los dos componentes anteriores, es decir, se observa una relación importante con respecto del rendimiento académico.

No sucede lo mismo con los componentes emocionales intrapersonal e interpersonal, respecto de los cuales no se encontró relación significativa con la variable rendimiento académico, es decir, parece no existir mucha conexión entre la capacidad de autoconocimiento y, por otro lado, la capacidad para establecer relaciones interpersonales con aquello que llamamos rendimiento académico.

No se trata de que no sean útiles para un mejor aprovechamiento o aprendizaje, sino que son factores que aún no han sido adecuadamente advertidos o profundizados por muchos estudiantes y docentes.

Aun para muchos maestros, habilidades sociales como la empatía o el liderazgo no tienen nada que ver con la experiencia del aprendizaje. Este tipo de creencias conduce a que en la práctica 
se valoren más los elementos netamente racionales, en detrimento de los componentes emocionales, que son decisivos para un aprendizaje real y significativo.

\section{CONCLUSIONES}

- La inteligencia emocional y el rendimiento académico de los estudiantes de la Facultad de Educación y Humanidades de la Universidad Alas Peruanas muestran tan solo cierto grado de asociación. Es decir, no existe una relación significativa entre ambas variables.

- Esta situación es debido a que en la praxis la variable dependiente rendimiento académico posee un contenido netamente racional; es decir, aquello que llamamos rendimiento académico es el puntaje numérico en función del número de respuestas bien contestadas en un examen, práctica o demostración. Esto ya ha sido advertido por numerosos investigadores: aquellos que brillaron en la vida académica no siempre alcanzaron lo mismo en la vida del trabajo, de familia o de pareja.

- En función de los resultados presentados, se puede concluir que existe una relación significativa entre el cociente emocional del estado de ánimo general y el rendimiento académico en estudiantes de la Facultad de Educación y Humanidades de la Universidad Alas Peruanas.

- Existe una relación significativa entre el cociente emocional del manejo del estrés y el rendimiento académico en los estudiantes de la Facultad de Educación y Humanidades de la Universidad Alas Peruanas.

- Del mismo modo, se encuentra relación significativa entre el cociente emocional de adaptabilidad y el rendimiento académico en estudiantes de la Facultad de Educación y Humanidades de la Universidad Alas Peruanas.
- Con respecto del grado de asociación del cociente emocional interpersonal y rendimiento académico de los estudiantes de la Facultad de Educación y Humanidades de la Universidad Alas Peruanas, se encuentra escasa relación entre las variables.

- Sucede lo mismo con respecto del cociente emocional intrapersonal y el rendimiento académico de los estudiantes de la Facultad de Educación y Humanidades de la Universidad Alas Peruanas, entre los cuales no se encontró una relación significativa.

\section{REFERENCIAS BIBLIOGRÁFICAS}

1. Gardner H. Estructura de la mente. La teoría de las inteligencias múltiples. Fondo de Cultura Económica, México, 1994.

2. Salovey P.y Mayer J.D.Emotional intelligence en imagination, cognition and personality. Cambridge University, 1990.

3. Goleman David. Inteligencia emocional. Edit. Javier Vergara. Buenos Aires, 1996.

4. Albunada Ruiz. Principios y procedimientos evaluativos. Ministerio de Educación, Chile, 1990.

5. Aliaga Pacora Alicia A.Inteligencia emocional de las docentes de educación inicial del distrito de Los Olivos. Tesis para optar el grado académico de maestro en Administración de la Educación. Escuela de Postgrado de la Universidad Inca Garcilaso de la Vega, Lima, 2004.

6. Arruda J.Didáctica y práctica de la enseñanza. Mc Graw-Hill, Bogotá, 1986.

7. Ávila Acosta R. B. Metodología de la Investigación. Estudios y Ediciones S. A., Lima, 2001.

8. Brockert S. y Braun G. Los tests de la inteligencia emocional. Robinbook, Barcelona, 1997. 
9. Bar-On R. The development of a concept de psychological well-being. Unpublished doctoral dissertation. Rhodes University, South Africa, 1997.

10. Chabot Daniel. Cultive su inteligencia emocional. Edic. Mensajero. Bilbao, 2001.

11. DíazPeñaElizabeth.Programade Motivación del Aprendizaje y su Efecto en el Rendimiento Académico en la Asignatura de Medicina Legal, Deontología y Ética Profesional en estudiantes del VIII ciclo de la Escuela Profesional de Obstetricia de la Universidad Privada Antenor Orrego de Trujillo 2000-I. Tesis para optar el grado de maestro en Investigación y Docencia Universitaria. Universidad Inca Garcilaso de la Vega, Lima, 2000.

12. Garcia Ampudia Lupe. Desarrollo afectivo y valorativo. Universidad Nacional Mayor de San Marcos, 2da. edic., Lima, 1999.

13. Hernández Sampieri Roberto. Metodología de la investigación. Mc Graw Hill, 2da. edic., México, 1999.

14. Qwisgaard Álvarez Jacinto. Inteligencia emocional: evaluación y desarrollo. Tesis para optar el grado de doctor en psicología. Universidad Inca Garcilaso de la Vega, Lima, 2001.

15. Ministerio de Educación. Crecer hacia un Sistema Nacional de Evaluación de
Rendimiento. Dirección Regional de Educación de La Libertad, Trujillo, 2000.

16. Salkind Neil J. Métodos de investigación. 3ra. Edic., Prentice May, México, 1999.

17. Shapiro L. La inteligencia emocional en los niños. Edit. Javier Vergara, Buenos Aires, 1988.

18. Steiner C. La educación emocional. Edit. Javier Vergara, Lima, 1987.

19. Sternberg R. J. Inteligencia exitosa. Cómo una inteligencia práctica y creativa determina el éxito en la vida. Paidós, Barcelona, 1997.

20. Tafur Portilla Raúl. La tesis universitaria. Edit. Mantaro, Lima, 1994.

21. Trianes Torres Victoria. Psicología de la educación para profesores. Reimpresión de la 1 ra. edic., Pirámide, España, 1996.

22. Ugarriza N. Evaluación de la inteligencia emocional a través del inventario de BarOn (I-CE) en una muestra de Lima Metropolitana. Edit. Libro Amigo, Lima, 2001.

23. Zegarra Tejada Dora. Personalidad, inteligencia emocional y estrés en los postulantes en el examen de admisión a la universidad y sus resultados. Tesis para optar el grado de Doctor en Psicología. Universidad Inca Garcilaso de la Vega, Lima, 2004. 